[7] has eonjectured that

$$
\ln p(g) \sim \sqrt{ } g .
$$

The maximum difference observed is 300 , whereas in this region a gap of 1040 may exist.

(e) Largest pair: The largest observed pair is

$$
1000000000149342 \pm 1 .
$$

Acknowledgement. We wish to thank the referee for his suggestions as to the presentation of the above data.

Department of Mathematics

Memorial University of Newfoundland

St. John's, Newfoundland

1. D. H. Lehmer, "Tables concerning the distribution of primes up to 37 millions," 1957 copy deposited in the UMT File \& reviewed in MTAC, v. 13, 1959, pp. 56-57.

2. G. H. HARDY \& J. E. LitTLEWOod, "Some problems of 'partitio numerorum'; III: On the expression of a number as a sum of primes," Acta Math. v. 44, 1923, pp. 1-70.

3. C. L. Baker \& F. J. Gruenberger, The First Six Million Prime Numbers, The Rand Corporation, Santa Monica, published by the Microcard Foundation, Madison, Wisconsin, 1959. Reviewed in Math. Comp., v. 15, 1961, p. 82, RMT4.

4. F. Gruenberger \& G. Armerding, "Statistics on first six million prime numbers," Paper P-2460 of the Rand Corporation, Santa Monica, 1961, 145 pp., $8 \frac{1}{2} \times 11$ in. Copy deposited in UMT File \& reviewed in Math. Comp., v. 19, 1965, pp. 503-505.

5. G. G. AlWAY, "A method of factorisation using a high-speed computer," MTAC, v. 6, no. 37,1952 , pp. $59-60$

6. D. N. Lehmer, List of Prime Numbers from 1 to 10,006,721, Hafner, New York, 1956.

7. D. Shanks, "On maximal gaps between successive primes," Math. Comp., v. 18, 1964, pp. $646-650$. MR $29 * 4745$.

\title{
A Note on Expansions Involving Meijer's $G$-Functions
}

\section{By Arun Verma}

1. Introduction. The expansions of Meijer's $G$-functions in a series of similar functions and their products with terminating hypergeometric functions, have been studied by several mathematicians as for example Meijer [6], Wimp and Luke [10] and others. It has been shown by the author [7] that these expansions can be written out easily from the known expansions of elementary functions by using induction through Laplace transform and its inverse. However, it is strange to notice that there is not even a single known expansion of Meijer's $G$-function in a series of product of $G$-functions. Also recently, the author [8], [9] has obtained the expansions of $G$-functions of two variables (defined by Agarwal [1]) in a series of similar functions and in a series of products of $G$-functions of two variables and terminating hypergeometric functions. In this paper, using the Laplace transform and its inverse, expansions of Meijer's $G$-function and its extension in a series of products of similar functions are obtained. The results given are

Received June 8, 1966. 
just to illustrate the method and obviously a variety of similar results could be multiplied.

2. Notations. Let

$$
[a]_{n}=a(a+1)(a+2) \cdots(a+n-1), \quad[a]_{0}=1,
$$

then the generalised hypergeometric function ${ }_{r} F_{8}$ is defined as

(1) ${ }_{r} F_{s}\left[\begin{array}{c}\left(a_{r}\right) ; z \\ \left(b_{s}\right)\end{array}\right]=\sum_{n=0}^{\infty} \frac{\left[\left(a_{r}\right)\right]_{n}}{[1]_{n}\left[\left(b_{s}\right)\right]_{n}} z^{n}, \quad r<s+1$ or $\quad r=s+1,|z|<1$,

where $\left(\alpha_{M, N}\right)$ means $(N-M+1)$ parameters $\alpha_{M}, \alpha_{M+1}, \cdots, \alpha_{N}$, but when $M=1$, then instead of writing $\left(\alpha_{1, N}\right)$ we write simply $\left(\alpha_{N}\right)$.

The Meijer's $G$-function is defined as

$$
G_{p, q}^{m, n}\left(z \mid \begin{array}{l}
\left(a_{p}\right) \\
\left(b_{q}\right)
\end{array}\right)=\frac{1}{2 \pi i} \int_{C} \Gamma\left[\begin{array}{c}
\left(b_{m}\right)-\xi, 1-\left(a_{n}\right)+\xi ; \\
\left(a_{n+1, p}\right)-\xi, 1-\left(b_{m+1, q}\right)+\xi
\end{array}\right] z^{\xi} d \xi
$$

$0 \leqq m \leqq q, 0 \leqq n \leqq p, p+q<2(m+n),|\arg z|<\frac{1}{2} \pi[-p-q+2(m+n)]$, where $C$ is a suitable contour. For details see Erdelyi [5] and

$$
\Gamma\left[\begin{array}{c}
\left(a_{p}\right),\left(b_{q}\right) ; \\
\left(c_{r}\right),\left(d_{s}\right)
\end{array}\right]=\frac{\Gamma\left[a_{1}\right] \Gamma\left[a_{2}\right] \cdots \Gamma\left[a_{p}\right] \Gamma\left[b_{1}\right] \Gamma\left[b_{2}\right] \cdots \Gamma\left[b_{q}\right]}{\Gamma\left[c_{1}\right] \Gamma\left[c_{2}\right] \cdots \Gamma\left[c_{r}\right] \Gamma\left[d_{1}\right] \Gamma\left[d_{2}\right] \cdots \Gamma\left[d_{s}\right]} .
$$

Lastly the $G$-function of two variables defined recently by Agarwal [1] is*

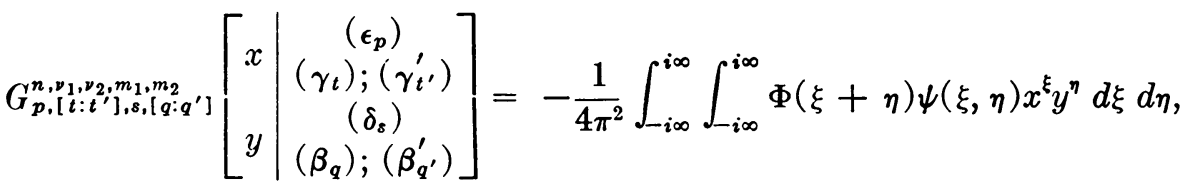

$$
\begin{aligned}
& \psi(\xi, \eta)=\Gamma\left[\begin{array}{c}
\left(\beta_{m_{1}}\right)-\xi,\left(\gamma_{\nu_{1}}\right)+\xi,\left(\beta_{m_{2}}^{\prime}\right)-\eta,\left(\gamma_{\nu_{2}}^{\prime}\right)+\eta \\
1-\left(\beta_{m_{1}+1, q}\right)+\xi, 1-\left(\gamma_{\nu_{1}+1, t}\right)-\xi, \\
1-\left(\beta_{m_{2}+1, q^{\prime}}^{\prime}\right)+\eta, 1-\left(\gamma_{v_{2}+1, t^{\prime}}^{\prime}\right)-\eta
\end{array}\right], \\
& \Phi(\xi+\eta)=\Gamma\left[\begin{array}{c}
1-\left(\epsilon_{n}\right)+\xi+\eta ; \\
\left(\epsilon_{n+1, p}\right)-\xi-\eta,\left(\delta_{s}\right)+\xi+\eta
\end{array}\right],
\end{aligned}
$$

where $0 \leqq m_{1} \leqq q, 0 \leqq m_{2} \leqq q^{\prime}, 0 \leqq \nu_{1} \leqq t, 0 \leqq \nu_{2} \leqq t^{\prime}, 0 \leqq n \leqq p$.

The sequence of parameters $\left(\beta_{m_{1}}\right),\left(\beta_{m_{2}}^{\prime}\right),\left(\gamma_{v_{1}}\right),\left(\gamma_{\nu_{2}}^{\prime}\right)$ and $\left(\epsilon_{n}\right)$ is chosen such that none of the poles of the integrand coincide. The paths of integration are indented, if necessary, in such a manner that all the poles of

$$
\Gamma\left[\left(\beta_{m_{1}}\right)-\xi\right] \text { and } \Gamma\left[\left(\beta_{m_{2}}^{\prime}\right)-\eta\right]
$$

lie to the right and those of $\Gamma\left[\left(\gamma_{\nu_{1}}\right)+\xi\right], \Gamma\left[\left(\gamma_{\nu_{2}}^{\prime}\right)+\eta\right]$ and $\Gamma\left[1-\left(\epsilon_{n}\right)+\xi+\eta\right]$ lie to the left of the imaginary axis.

The integral (2.3) is convergent if

$$
\begin{aligned}
p+q+s+t & <2\left(m_{1}+\nu_{1}+n\right), \\
p+q^{\prime}+s+t^{\prime} & <2\left(m_{2}+\nu_{2}+n\right)
\end{aligned}
$$

* It may be mentioned that the $G$-function of two variables which we are taking is a slight variant of that given by Agarwal [1], though in essence the function is the same. 
and

$$
\begin{aligned}
& |\arg x|<\pi\left[m_{1}+\nu_{1}+n-\frac{1}{2}(p+q+s+t)\right] \\
& |\arg y|<\pi\left[m_{2}+\nu_{2}+n-\frac{1}{2}\left(p+q^{\prime}+s+t^{\prime}\right)\right]
\end{aligned}
$$

3. Cooke [4] has shown that

$$
\begin{aligned}
\sum_{m=1}^{\infty}\left(\frac{1}{2} m x\right)^{-\mu} J_{\mu}(m x)\left(\frac{1}{2} m y\right)^{-\nu} J_{\nu}(m y) & \\
= & -\frac{1}{2 \Gamma[\mu+1, \nu+1]}+\frac{\pi^{1 / 2}}{y \Gamma\left[\mu+1, \nu+\frac{1}{2}\right]} \\
& \times{ }_{2} F_{1}\left[\frac{1}{2}-\nu, \frac{1}{2} ; \mu+1 ; \frac{x^{2}}{y^{2}}\right], \quad \pi>y>x>0, \mu, \nu>-\frac{1}{2}
\end{aligned}
$$

In the notation of $G$-functions (3.1) can be rewritten as

$$
\begin{aligned}
2 \sum_{n_{0}=1}^{\infty} G_{2,0}^{0,1}\left(\frac{x}{m^{2}} \mid 1,1+\mu\right) G_{2,0}^{0,1}\left(\frac{y}{m^{2}} \mid\right. & 1,1+\nu)=\Gamma[; 1+\nu, 1+\mu] \\
& +\frac{y^{1 / 2}}{\pi} \sin \pi\left(\nu+\frac{1}{2}\right) G_{2,2}^{1,2}\left(-\frac{x}{y} \mid \begin{array}{c}
1,1+\mu \\
\frac{1}{2}-\nu, \frac{1}{2}
\end{array}\right)
\end{aligned}
$$

From this we deduce the following general expansion

$$
\begin{aligned}
& 2 \sum_{m=1}^{\infty} G_{2+p, q}^{r, s+1}\left(\frac{x}{m^{2}} \mid \begin{array}{l}
1,\left(a_{p}\right), 1+\mu \\
\left(b_{q}\right)
\end{array}\right) G_{2+P, Q}^{R, s+1}\left(\frac{y}{m^{2}} \mid \begin{array}{l}
1,\left(\alpha_{P}\right), 1+\nu \\
\left(\beta_{Q}\right)
\end{array}\right) \\
& =-\Gamma\left[\begin{array}{l}
\left(b_{r}\right), 1-\left(a_{s}\right),\left(\beta_{S}\right), 1-\left(\alpha_{R}\right) ; \\
1+\nu, 1+\mu, 1-\left(b_{r+1, q}\right),\left(a_{s+1, p}\right), 1-\left(\beta_{R+1, Q}\right),\left(\alpha_{S+1, P}\right)
\end{array}\right] \\
& +\frac{y^{1 / 2}}{\pi} \sin \pi\left(\nu+\frac{1}{2}\right) G_{2+p+Q, 2+q+P}^{1+r+s, 2+s+R}\left(-\frac{x}{y} \mid \begin{array}{l}
1,\left(a_{r}\right), \frac{3}{2}-\left(\beta_{Q}\right),\left(a_{r+1, p}\right), 1+\mu \\
\frac{1}{2}-\nu, \frac{1}{2},\left(b_{s}\right), \frac{3}{2}-\left(\alpha_{P}\right),\left(b_{s+1, q}\right)
\end{array}\right),
\end{aligned}
$$

provided $\pi>y>x>0, \mu, \nu>-\frac{1}{2}, 0 \leqq r \leqq q, 0 \leqq s \leqq p, 0 \leqq R \leqq Q$, $0 \leqq S \leqq P, p+q<2(r+s), P+Q<2(R+S)$ and

$$
\begin{aligned}
& |\arg x|<\frac{1}{2} \pi[2(r+s)-p-q], \\
& |\arg y|<\frac{1}{2} \pi[2(R+S)-P-Q],
\end{aligned}
$$

and the series of products of $G$-functions on the right has a meaning.

We prove this result by the method of finite mathematical induction. To do so, we suppose that this result holds for certain values of $r, s, p, q, R, S, P, Q$. Replace $x$ by $x / t$ on both sides, multiply both sides by $t^{b-1}$ and take the Laplace transform with respect to " $t$ ". Using the result

$$
\int_{0}^{\infty} e^{-t} t^{\gamma-1} d t=\mathrm{\Gamma}[\gamma], \quad \mathrm{Rl} \gamma>0,
$$

we find (3.3) with $r$ replaced by $[r+1] .{ }^{*}$ This completes the induction with respect to " $r$ ". Similarly to complete induction with respect to " $p$ ", replace $x$ by $x t$

* The restrictions on the parameters arise due to the particular method followed and can be waived off by analytic continuation. 
and multiply both sides of (3.3) by $t^{-\delta}$. Then taking the inverse Laplace transform with respect to " $t$ " and using the result

$$
\int_{c} e^{t} t^{-\gamma} d t=\frac{1}{\Gamma[\gamma]}, \quad \operatorname{Rl} \gamma>0,
$$

we find the relation (3.3) with $p$ replaced by $[p+1]$. Similarly, the induction with respect to $s, q, R, S, P$ and $Q$ can be completed. But for $r=0=s=p=$ $q=R=S=P=R$, (3.3) reduces to (3.2) and this completes the proof of (3.3), by induction.

On the other hand, if we start from the following result due to Buchholz [2]:

$$
\sum_{m=1}^{\infty} \frac{J_{\nu}\left(x \gamma_{\nu, n}\right) J_{\nu}\left(x \gamma_{\nu, n}\right)}{\left[J_{\nu+1}\left(\gamma_{\nu, n}\right)\right]^{2}\left(z^{2}-\gamma_{\nu, n}^{2}\right)}=\frac{\pi J_{\nu}(x z)}{4 J_{\nu}(z)}\left[J_{\nu}(z) Y_{\nu}(X z)-J_{\nu}(X z) Y_{\nu}(z)\right],
$$

$0 \leqq x \leqq X \leqq 1$, and $\nu$ is not an integer, the zeros of $z^{-\nu} J_{\nu}(z)$ being arranged in the ascending magnitude of $\operatorname{Rl}\left(\gamma_{v, n}\right)>0$, are $\gamma_{v, n}(n=0,1,2, \cdots)$, and we make use of the Laplace transform and its inverse, to get the following very general result:

$$
\begin{aligned}
& \sum_{n=1}^{\infty} \frac{\gamma_{\nu, n}^{2} G_{2+r, s}^{p, q+1}\left(\frac{x}{\gamma_{\nu, n}^{2}} \mid \begin{array}{l}
1,\left(a_{r}\right), 1+\nu \\
\left(b_{s}\right)
\end{array}\right) G_{2+R, S}^{P, Q+1}\left(\frac{x}{\gamma_{\nu, n}^{2}} \mid \begin{array}{l}
1,\left(\alpha_{R}\right), 1+\nu \\
\left(\beta_{S}\right)
\end{array}\right)}{\left[J_{r+1}\left(\gamma_{\nu, n}\right)\right]^{2}\left(z^{2}-\gamma_{\nu, n}^{2}\right)} \\
& =\pi z^{2 \nu} G_{2+r, s}^{p, q+1}\left(\frac{x}{z^{2}} \mid \begin{array}{l}
1,\left(a_{r}\right), 1+\nu \\
\left(b_{s}\right)
\end{array}\right)\left[\begin{array}{ll}
\cot \pi \nu J_{\nu}(z) & (z)
\end{array}\right. \\
& \left.-Y_{\nu}(z)\right\} G_{2+R, S}^{P, Q+1}\left(\frac{x}{z^{2}} \mid \begin{array}{l}
1,\left(\alpha_{R}\right), 1+\nu \\
\left(\beta_{S}\right)
\end{array}\right) \\
& \left.-\operatorname{cosec} \pi \nu G_{2+R, S}^{P, Q+1}\left(\frac{x}{z^{2}} \mid \begin{array}{l}
1+\nu,\left(\alpha_{R}\right), 1 \\
\left(\beta_{S}\right)
\end{array}\right)\right],
\end{aligned}
$$

where

$0 \leqq x \leqq X \leqq 1, \quad 0 \leqq q \leqq r, \quad 0 \leqq P \leqq S, \quad 0 \leqq Q \leqq R$,

$$
0 \leqq p \leqq s, \quad r+s<2(p+q), \quad R+S<2(P+Q),
$$

$\left|\arg 1 / z^{2}\right|<\frac{1}{2} \pi[2(r+s)-p-q], \quad\left|\arg 1 / z^{2}\right|<\frac{1}{2} \pi[2(R+S)-P-Q]$ and the series of products of $G$-functions on the left-hand side has a meaning.

4. In this section, we proceed to find the expansions of $G$-functions of two variables in a series of products of two $G$-functions. To prove such a result we start from a known result due to Burchnall and Chaundy [3, Eq. 26]

$$
\begin{aligned}
& F^{(2)}\left[a ; b, b^{\prime} ; c, c^{\prime} ; x, y\right]=\sum_{r=0}^{\infty} \frac{[a]_{r}[b]_{r}\left[b^{\prime}\right]_{r}}{[1]_{r}[c]_{r}\left[c^{\prime}\right]_{r}} \\
& \cdot x^{r} y^{r} F\left[\begin{array}{c}
a+r, b+r ; x \\
c+r
\end{array}\right] F\left[\begin{array}{c}
a+r, b^{\prime}+r ; y \\
c^{\prime}+r
\end{array}\right], \quad|x|+|y|<1,
\end{aligned}
$$

where $F^{(2)}\left[a ; b, b^{\prime}, c, c^{\prime} ; x, y\right]$ is the Appell's second function and is defined as

$$
\sum_{m=0}^{\infty} \sum_{n=0}^{\infty} \frac{[a]_{m+n}[b]_{m}\left[b^{\prime}\right]_{n}}{[1]_{m}[1]_{n}[c]_{m}\left[c^{\prime}\right]_{n}} x^{m} y^{n}
$$


Then rewriting (4.1) in terms of $G$-functions of two and one variables using $[5,5.6(1)]$ and $[1,3-$ iv], respectively, we get

$$
\begin{aligned}
& G_{1,[1: 1], 0,[2: 2]}^{1,1,1,1,1}\left[\begin{array}{l}
x+c^{\prime}-a-1 \\
1+b-c, 1+b^{\prime}-c^{\prime} \\
\cdots \cdots \cdots \cdots \cdots \cdots \cdots \\
c-1,0 ; c^{\prime}-1,0
\end{array}\right]=\sum_{r=0}^{\infty} \frac{e^{\left(c+c^{\prime}\right) \pi i}}{r ! \Gamma[a+r]} \\
& \times G_{2,2}^{2,1}\left(\frac{1}{x} \mid \begin{array}{l}
c-r, 2 c-1 \\
a+c-1, b+c-1
\end{array}\right) \\
& \cdot G_{2,2}^{2,1}\left(\frac{1}{y} \mid \begin{array}{l}
c^{\prime}-r, 2 c^{\prime}-1 \\
a+c^{\prime}-1, b^{\prime}+c^{\prime}-1
\end{array}\right), \quad|x|+|y|<1 .
\end{aligned}
$$

Then using the Laplace transform and its inverse, we can generalise (4.2) to the result:

$$
\begin{aligned}
& G_{1,[1+P: 1+Q], 0,[2+R: 2+r]}^{1,1+p, 1+q, 1+r, 1+t}\left[x \mid \begin{array}{l}
c+c^{\prime}-a-1 \\
1+b-c,\left(a_{P}\right) ; 1+b^{\prime}-c^{\prime},\left(b_{Q}\right) \\
\ldots \ldots \ldots \ldots \cdots \cdots \cdots \cdots \cdots \cdots \cdots \cdots \cdots \cdots \cdots \\
c-1,\left(c_{R}\right), 0 ; c^{\prime}-1,\left(d_{T}\right), 0
\end{array}\right]=\sum_{r=0}^{\infty} \frac{e^{\left(c+c^{\prime}\right) \pi i}}{r ! \Gamma[a+r]} \\
& \cdot G_{2+\mathrm{T}, 2+Q}^{1+a+t}\left(y \mid \begin{array}{l}
2-a-c^{\prime}, 2-b-c^{\prime},\left(d_{T}\right) \\
1-c^{\prime}+r,\left(b_{Q}\right), 2-2 c^{\prime}
\end{array}\right) \\
& \cdot G_{2+R, 2+P}^{1+p .2+r}\left(x \mid \begin{array}{l}
2-c-a, 2-b-c,\left(c_{R}\right) \\
1-c+r,\left(a_{p}\right), 2-2 c
\end{array}\right),
\end{aligned}
$$

where

$$
\begin{aligned}
& |x|+|y|<1, \quad 0 \leqq p \leqq P, \quad 0 \leqq q \leqq Q, \quad 0 \leqq r \leqq R, \\
& 0 \leqq t \leqq T, \quad P+R<2(1+r+p), \quad Q+T<2(1+q+t), \\
& |\arg x|<\frac{1}{2} \pi[2(1+r+p)-P-R], \\
& |\arg y|<\frac{1}{2} \pi[2(1+q+t)-T-Q]
\end{aligned}
$$

and the series on the right hand side has a meaning. This contains $[3,92]$ as a special case.

Similar expansions can be obtained if one starts from [3, 28] and [3,30]. But if one starts from the expansions [3, 27], [3, 29], [3, 31], due to Burchnall and Chaundy, expansions of products of two $G$-functions in a series of $G$-functions of two variables, can be written.

Department of Mathematics

The University

Gorakhpur, India

1. R. P. Agarwal, "An extention of Meijer's G-function”, Proc. Nat. Inst. Sci. India. Sect. A, v. 32, 1965.

2. H. BUCHHOLZ, "Bemerkungen zu einer Entwicklungsformel aus der Theorie der Zylinderfunktionen", $Z$. Angew. Math. Mech., v. 25/27, 1947, pp. 245-252. MR 9, 282.

3. J. L. BuRChNali \& T. W. CHAUNDY, "Expansions of Appell's double hypergeometric functions", Quart. J. Math. (Oxford), v. 11, 1940, pp. 249-270. MR 2, 287.

4. R. G. Cooke, Proc. London. Math. Soc., v. 28, 1928, pp. 207-241.

5. A. ERDELYI, ET AL., Higher Transcendental Functions, Vol. I, McGraw-Hill, New York, 1953. MR 15, 419 . 
6. C. S. Meijer, "Expansion theorems for the G-functions. I-X," Indag. Math., v. 14, 1952, pp. $369-379$ and $483-487$; v. 15 , 1953, pp. 43-49, 187-193 and 349-357; v. 16, 1954, pp. 77-82, 83-91 and 273-279; v. 17, 1955, pp. 243-251 and 309-314. MR 14, 469; MR 14, 642; MR 14, 748; MR 14, 979; MR 15, 422; MR 15, 791; MR 15, 955; MR 16, 1106.

7. A. Verma, "A class of expansions of $G$-functions and the Laplace transform," Math. Comp., v. 19, 1965, pp. 661-664.

8. A. VERMA, "Expansions of hypergeometric functions of two variables", Math. Comp., v. $20,1966,590-596$.

9. A. Verma, "A note on an expansion of hypergeometric functions of two variables", Math. Comp., v. 20, 1966, 413-417.

10. J. WIMP \& Y. L. LUKe, "Expansion formulas for generalised hypergeometric functions", Rend. Circ. Mat. Palermo, (2), v. 11, 1962, pp. 351-366.

\section{Approximations for the Psi (Digamma) Function}

\section{By William T. Moody}

A series of approximations has been derived for the psi function. As used here, the psi function is defined as the derivative of the natural logarithm of the gamma function; that is

$$
\psi(x)=\frac{d[\ln \Gamma(x)]}{d x}=\frac{\Gamma^{\prime}(x)}{\Gamma(x)} .
$$

The approximations are best in the Chebyshev sense, in that the magnitude of the maximum error in the prescribed interval is minimized. Each approximation is of the form

wherein

$$
\psi(1+x)=\frac{x}{1+x}-\gamma+\frac{1}{2} x^{n+1}+\sum_{i=1}^{n} c_{i}\left(x^{i}-x^{n+1}\right)+\epsilon(x), \quad 0 \leqq x \leqq 1,
$$

$$
\gamma=0.5772 \cdots, \quad \text { (Euler's constant). }
$$

Values of the constants, $c_{i}$, and the limiting values of $\epsilon$ for $n=4,5,6$ are given in Table 1 below. The error of the approximation vanishes at the end points.

\begin{tabular}{|c|c|c|c|}
\hline$n \ldots \ldots$ & 4 & 5 & 6 \\
\hline$\epsilon<$ & $1.3 \times 10^{-6}$ & $1.3 \times 10^{-7}$ & $1.3 \times 10^{-8}$ \\
\hline$i$ & \multicolumn{3}{|c|}{$c_{i}$} \\
\hline $\begin{array}{l}1 \\
2 \\
3 \\
4 \\
5 \\
6\end{array}$ & $\begin{array}{c}+0.644876 \\
-0.201186 \\
+0.077968 \\
-0.026867 \\
-\end{array}$ & $\begin{array}{c}+0.6449266 \\
-0.2019040 \\
+0.0812656 \\
-0.0334532 \\
+0.0111653\end{array}$ & $\begin{array}{r}+0.64493313 \\
-0.20203181 \\
+0.08209433 \\
-0.03591665 \\
+0.01485925 \\
-0.00472050\end{array}$ \\
\hline
\end{tabular}

TABLE 1

Values of Constants

Bureau of Reclamation

Federal Center

Denver, Colorado

Received March 28, 1966. Revised August 4, 1966. 